\title{
Expression of genes encoding cytotoxic cell-associated serine proteases in thymocytes
}

\author{
Werner Held, H. Robson MacDonald ${ }^{\mathbf{1}}$, and Christoph Mueller \\ Department of Pathology, University of Bern, Freiburgstrasse 30, CH-3010 Bern, Switzerland \\ 1 Ludwig Institute for Cancer Research, Lausanne Branch, CH-1066 Epalinges, Switzerland
}

Key words: granzymes, in situ hybridization, interleukin 2 receptor, perforin, thymus

\begin{abstract}
A family of homologous serine esterases designated granzyme $\mathrm{A}-\mathrm{H}$ and the pore-forming protein perforin are present in cytoplasmic granules of mature peripheral cytolytic $T$ lymphocytes and natural killer cells. In vivo, the majority of cytotoxic T cells containing these granuleassociated proteins are of the $\mathrm{CD} 4-\mathrm{CD} 8+$ phenotype. It is generally assumed that these cells are derived from immature CD4-CD8 - thymocytes. However, the precise intrathymic differentiation steps leading to functionally mature cytotoxic $T$ cells are unclear. Thus we decided to analyze the expression of genes in the thymus which are preferentially expressed in mature cytotoxic cells, i.e. granzyme A, granzyme B, and perforin. In situ hybridization on tissue sections revealed the expression of genes coding for granzyme $A$ and granzyme $B$ in the thymus. No evidence was found, however, for thymocytes expressing the perforin gene. Granzyme A and granzyme B mRNA positive cells in the thymus are almost exclusively CD4-CD8- thymocytes, particularly of the CD3- IL2R- phenotype.
\end{abstract}

\section{Introduction}

Large cytoplasmic granules of cytotoxic T lymphocytes (CTL) and natural killer (NK) cells contain among other molecules a family of highly homologous serine proteases, designated granzyme $A-H(1)$ and the pore-forming protein perforin (2). Purified perforin shows cytolytic activity against red blood cells and certain cell lines (3). The precise role of the serine proteases in cellmediated killing, however, is not known. Inhibition studies with protease inhibitors including the synthetic inhibitor H-D-Pro-PheArg-chloromethyl-ketone, which selectively inhibits granzyme $A$, indicated an important role for these proteases in cell-mediated cytolysis $(4,5)$. However, no direct cytotoxic effects of purified granzyme A against various normal and tumor target cells were found (6), implying a more indirect role for this protease in cellmediated cytolysis. Other functions, not directly related to cellmediated cytotoxicity, such as an involvement in $B$ cell activation or degradation of extracellular matrix (7), were also proposed for serine proteases in T lymphocytes. In vivo, the majority of the perforin- and granzyme-containing CTL seem to be of the CD4-CD8+ phenotype $(8-11)$, although a minor fraction of Thy-1+CD4+CD8- peripheral $T$ cells also express the granzyme A gene in vivo (8).

It is generally assumed that peripheral $C D 4+C D 8-$ and CD4-CD8 + $T$ cells differentiate from immature CD4-CD8- thymocytes, the so-called double negative thymocytes (DN), via a $\mathrm{CD} 4+\mathrm{CD} 8+$ intermediate. The intrathymic differentiation step(s) leading to functionally mature cytotoxic $T$ cells are unclear (for review see ref. 12). We decided to address this question by analyzing the expression of genes in the thymus that are preferentially associated with mature cytotoxic cells, i.e. granzyme A (1), granzyme B (1), and perforin (13).

\section{Methods}

Preparation of labeled RNA probes

A 775 bp cDNA fragment of the granzyme A gene [also designated HF and kindly provided by I. L. Weissman (14); this gene has also been named CTLA-3 (15), TSP-1 (6), BLT esterase (16), or serine esterase 1 (17)], a $1.1 \mathrm{~kb}$ fragment of the granzyme $\mathrm{B}$ gene [also designated CCP-1/C11 and kindly provided by R. C. Bleackley (18); this gene has also been named CTLA-1 (15) or serine esterase $2(17)]$, a 521 bp cDNA fragment of the perforin gene (kindly provided by $\mathrm{H}$. Hengartner, 13) and a $1.1 \mathrm{~kb}$ fragment of the IL2R p55 chain gene $(19$, kindly provided by D. Pardoll, Bethesda) were subcloned into the transcription vector pGEM 1 using standard techniques. After linearization of the 


\section{Expression of serine protease genes in the thymus}

plasmids with the appropriate restriction endonucleases, $35 \mathrm{~S}$ labeled antisense and sense RNA probes were prepared using T7 and SP6 RNA polymerases, respectively, as previously described in detail (8).

\section{In situ hybridization}

In situ hybridization was performed on $5 \mu \mathrm{m}$ thick frozen sections of the thymus from 6 - to 8-week-old C57BL/Ka or BALB/c mice as previously described in detail (8). In brief, tissue sections were fixed in 4\% PBS-buffered paraformaldehyde for $20 \mathrm{~min}$ before proteinase $\mathrm{K}$ treatment. After post-fixation in paraformaldehyde and acetylation the hybridization was performed with $2 \times 10^{5}$ $\mathrm{cpm}{ }^{35}$ S-labeled RNA probe/ $\mu$ l of hybridization solution for $18 \mathrm{~h}$ at $48^{\circ} \mathrm{C}$. After digestion of single-stranded, non-hybridized RNA with RNase $A$ and $T 1$ and extensive washing, slides were dipped into NTB 2 nuclear track emulsion (Eastman Kodak). Hybridized slides were exposed for 10-20 days in a light-tight box at $-70^{\circ} \mathrm{C}$, developed and subsequently counterstained with nuclear fast red $(0.05 \%$ in $5 \%$ aluminium sulfate).

\section{Flow microfluorometry and cell sorting}

Total C57BL/6 thymocytes were stained with a mixture of FITCcoupled anti-CD8 mAb H35-17.2 (20) and phycoerythrin (PE)coupled anti-CD4 mAb GK-1.5 (21) and fixed immediately with $3 \%$ paraformaldehyde. The four major thymocyte subsets (CD4-CD8-, $\mathrm{CD} 4+\mathrm{CD} 8+, \mathrm{CD} 4+\mathrm{CD}^{-}$, and $\mathrm{CD} 4-\mathrm{CD} 8+$ ) were sorted according to the two-color fluorescence distribution.

For analysis of immature thymocyte subsets, CD4-CD8cells were first purified by treatment with a mixture of rat IgM mAbs RL172.4 (anti-CD4) and 3.168.1 (anti-CD8) in the presence of rabbit complement as described in detail elsewhere (22). CD4-CD8- thymocytes were further stained, fixed, and sorted according to their expression of IL-2 receptor (p55 chain) or CD3 using rat mAbs PC61.5 (23) and $17 A 2$ (G. Miescher and H. R. MacDonald, unpublished data), respectively. In most experiments, $\sim 10^{4}$ sorted cells were deflected directly onto pre-cleaned and poly-L-lysine-coated microscopic slides. In situ hybridization on sorted thymocytes was performed as described above for tissue sections.

\section{Evaluation of in situ hybridizations}

Tissue sections and cell preparations were hybridized with ${ }^{35} \mathrm{~S}$ labeled antisense RNA probes and, as a negative control, also with the respective sense probe which is identical to the cellular target mRNA. After hybridization with an antisense probe, cells were considered positive for gene expression when they had at least three times as many silver grains as cells hybridized with the corresponding sense RNA probe. For the assessment of the frequency of mRNA-positive cells, usually 2000-3000 isolated thymocytes were considered for evaluation.

\section{Results}

\section{Localization of gene expression in the adult thymus}

Frozen sections of the thymus from 6- to 8-week-old C57BL/Ka mice were hybridized in situ with ${ }^{35}$ S-labeled antisense RNA probes for the genes coding granzyme $A$, granzyme $B$, and perforin. Cells expressing granzymes $A$ and $B$ were regularly observed on thymus sections, whereas no evidence for
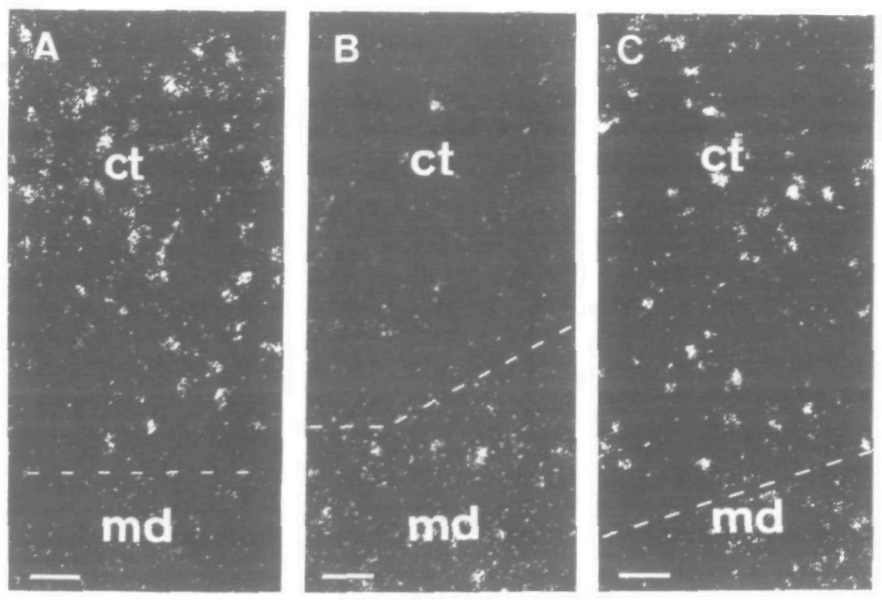

Fig. 1. Expression of the granzyme $A$, granzyme $B$, and IL2R genes in the thymus of a 6-week-old C57BL/Ka mouse. Dark-field photomicrographs show frozen sections of the thymus hybridized in situ with radiolabeled antisense RNA probes. Granzyme A gene expression is mainly observed scattered throughout the thymic cortex (ct) (A). Granzyme B-positive cells are predominantly found in the medullary area (md) of the thymus whereas cells containing IL2R mRNA are found in both the thymic cortex and the medullar $(C)$. Scale bars represent $50 \mu \mathrm{m}$ and the dashed line indicates the cortico-medullary junction.

transcription of the perforin gene was found. Cells expressing granzyme $A$ are almost exclusively located scattered throughout the thymic cortex, at slightly increased numbers in the subcapsular zone (Fig. 1A). They are much more frequent than granzyme B-positive cells which are predominantly found at a low frequency in the medullary area of the thymus (Fig. 1B).

The IL2R p55 chain is expressed on the cell surface of a distinct subset of early DN (23). Its expression in the thymus was previously characterized with the monoclonal anti-IL2R antibody PC61.5. Immunostainings with PC61.5 revealed the presence of positive cells scattered throughout the thymic cortex and medulla (23). We analyzed the expression of the IL2R gene in comparison to the IL2R surface expression to determine their correlation and as an internal control for the staining and sorting procedure. Cells containing IL2R mRNA were found in both the thymic cortex and the medulla (Fig. 1C). Generally, positive cells in the cortex showed a higher number of IL2R transcripts than positive cells in the medulla as was assessed by the number of silver grains over positive cells on tissue sections.

In order to determine whether the observed expression of granzyme $A$, granzyme $B$, perforin, and $I L 2 R$ is unique to the $\mathrm{C} 57 \mathrm{BL} / \mathrm{Ka}$ mouse strain we also hybridized thymus sections of $B A L B / C$ mice with the respective RNA probes. Granzyme A and IL2R gene expression was slightly more prominent in the subcapsular region of the thymus from BALB/C mice. However, the frequency and localization of mRNA-positive thymocytes did not differ significantly between BALB/C and C57BL/Ka mice (not shown).

Phenotype of thymocytes expressing the genes for granzyme $A$ and granzyme $B$

The frequency of thymocytes which are positive for the expression of a particular gene was determined by in situ hybridization on cytospins of thymocyte suspensions. About $1.3 \%$ of total 
Table 1. Phenotype of thymocytes expressing the genes for granzyme A and granzyme B

\begin{tabular}{|c|c|c|c|c|}
\hline \multirow[t]{2}{*}{ Thymic subset } & \multirow{2}{*}{$\begin{array}{l}\% \text { of total } \\
\text { thymocytes }\end{array}$} & \multicolumn{3}{|l|}{ Gene probe } \\
\hline & & Granzyme $A^{a}$ & Granzyme $\mathrm{B}^{\mathrm{a}}$ & IL $2 R^{a}$ \\
\hline Total thymocytes & 100 & $1.3 \pm 0.4$ & $+b$ & $1.5 \pm 0.4$ \\
\hline $\mathrm{CD} 4+\mathrm{CD} 8-$ & 10 & 0 & 0 & $0.5 \pm 0.2$ \\
\hline CD4-CD8 + & 4 & $1.5 \pm 0.6$ & 0 & 0 \\
\hline $\mathrm{CD} 4+\mathrm{CD} 8^{+}$ & 83 & 0 & 0 & 0 \\
\hline CD4-CD8- & 3 & $23.1 \pm 3.7$ & $2.2 \pm 0.6$ & $26.2 \pm 4.6$ \\
\hline
\end{tabular}

aPercentage of mRNA-positive cells in the respective thymocyte subsets. Data represent means \pm SD of three different experiments.

bPositive cells are occasionally observed.
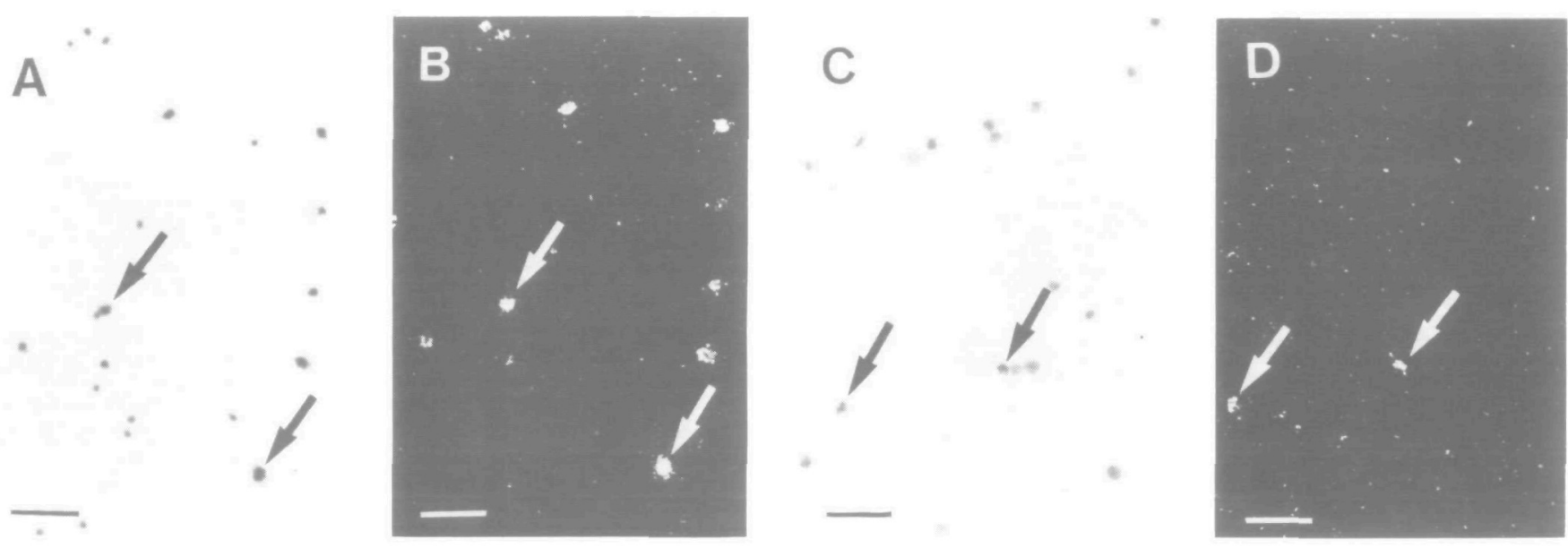

Fig. 2. Granzyme A gene expression in $\mathrm{CD} 4-\mathrm{CD} 8-\mathrm{CD} 3-\mathrm{IL} 2 \mathrm{R}-$ and $\mathrm{CD} 4-\mathrm{CD} 8-\mathrm{CD} 3^{-} \mathrm{IL} 2 \mathrm{R}^{+}$thymocytes. Thymocyte subpopulations were isolated and subsequently hybridized in situ at the single-cell level with the ${ }^{35}$ S-labeled antisense RNA probe of the granzyme A gene. The bright-field photomicrograph shows $\mathrm{CD} 4-\mathrm{CD} 8-\mathrm{CD} 3^{-} \mathrm{IL2R}$ - thymocytes (A) with a high percentage of granzyme $\mathrm{A}$ gene-expressing cells which are easily seen in the dark-field (B). Selected positive cells are arrowed. Only a few granzyme A mRNA-positive cells (arrowed) are found among CD4 - CD8$\mathrm{IL} 2 \mathrm{R}+$ thymocytes $(\mathrm{C}, \mathrm{D})$. Scale bars represent $50 \mu \mathrm{m}$.

thymocytes are positive for granzyme A mRNA, whereas granzyme $B$ positive cells are at least $5-10$ times less frequent. IL2R mRNA-positive cells represent $-1.5 \%$ of total thymocytes (Table 1). A slightly higher portion of thymocytes express IL2R protein on their surface (23).

For the phenotypic characterization of thymocytes expressing the granzyme A or granzyme B gene, thymocytes were first separated into the four major subsets, i.e. CD4+CD8- $(10 \%$ of total thymocytes), CD4-CD8+ (4\%), CD4+CD8+ (83\%), and CD4-CD8- $(3 \%)$, the so-called double negative thymocytes (DN), using a fluorescence-activated cell sorter (FACS). Separated thymocytes were subsequently hybridized at the single cell level with radiolabeled antisense RNA probes. Granzyme A, granzyme $B$, and IL2R mRNA-positive cells are almost exclusively found in the most immature of the four major thymic subsets, i.e. in DN. Very few CD4-CD8 + thymocytes (1.5\%) were also found to express the granzyme A gene (Table 1).

Recently an immature subpopulation of CD4-CD8+ thymocytes was identified which does not express the $T$ cell receptor (TCR)-associated CD3 complex (24). Therefore CD4-CD8 + thymocytes which contain $-1.5 \%$ granzyme $A$ mRNA-positive cells were further separated into a CD3+ $(70 \%$ of $\mathrm{CD} 4-\mathrm{CD} 8+$ ) and a CD3- (30\%) subset and subsequently
Table 2. Phenotype of CD4-CD8 + thymocytes expressing the gene for granzyme A

\begin{tabular}{llc}
\hline & $\begin{array}{l}\% \text { of total } \\
\text { thymocytes }\end{array}$ & Granzyme Aa \\
\hline CD4-CD8+CD3+ & 3 & 1.5 \\
CD4-CD8+ CD3 ${ }^{-}$ & 1 & 19.2 \\
\hline
\end{tabular}

aPercentage of mRNA-positive cells in the respective thymocyte subset. Data represent means of two different experiments.

hybridized with an antisense RNA probe of the granzyme A gene: mature thymocytes of the CD3+ CD4-CD8+ phenotype contain a very small fraction of granzyme A mRNA-positive cells $(1.5 \%)$, whereas $\sim 20 \%$ positive cells are found in the corresponding immature CD3- CD4-CD8+ subpopulation (Table 2).

Cells expressing genes for granzymes $A$ and $B$ in subsets of CD4-CD8-thymocytes

DN, which contain the precursors of all other major thymic subsets (25), are functionally and phenotypically very heterogeneous and account for only $2-4 \%$ of total cells in the thymus 
Table 3. Cells expressing genes for granzymes $A$ and $B$ in subsets of $C D 4-C D 8-$ thymocytes

\begin{tabular}{|c|c|c|c|c|c|}
\hline \multirow[t]{2}{*}{ Populationa } & \multirow[t]{2}{*}{ CD4-CD8- subset } & \multirow{2}{*}{$\begin{array}{l}\% \text { of } \\
\text { CD4-CD8- }\end{array}$} & \multicolumn{3}{|l|}{ Gene probeb } \\
\hline & & & Granzyme A & Granzyme B & IL2R \\
\hline & total CD4-CD8- & 100 & 25.0 & 2.1 & 28.7 \\
\hline A & $\mathrm{IL} 2 \mathrm{R}-\mathrm{CD} 3^{-}$ & 30 & 48.1 & 2.8 & 5.5 \\
\hline$B$ & $\mathrm{IL} 2 \mathrm{R}+\mathrm{CD} 3-$ & 45 & 7.8 & 1.0 & 68.4 \\
\hline $\mathrm{C} / \mathrm{D}$ & $\mathrm{IL} 2 \mathrm{R}-\mathrm{CD} 3+$ & 25 & 29.6 & 2.6 & 1.0 \\
\hline
\end{tabular}

aNomenclature according to ref. 12

bPercentage of mRNA-positive cells in the respective thymocyte subset. Data represent means of two different experiments.

(22). They can be divided into subpopulations according to the surface expression of the IL2R and the CD3 antigen: population A consists of DN IL2R-CD3- thymocytes ( $30 \%$ of DN), which are enriched for rapidly cycling thymocytes. Population $\mathrm{B}$ includes DN IL2R + CD3 - thymocytes ( $45 \%$ of DN). Populations $\mathrm{C} / \mathrm{D}$ are DN IL2R-CD3+ thymocytes (25\% of DN); the CD3 antigen is associated with a functional TCR of either the $\gamma / \delta$ (population $\mathrm{C}$ ) or $\alpha / \beta$ type [population $\mathrm{D}$; for review see MacDonald et al. (12)].

The distribution of cells expressing genes for granzymes $A$ and $B$ among DN thymocytes was determined by separating the DN subsets by FACS and subsequent in situ hybridization with RNA probes. About $8 \%$ of IL2R-positive cells (population B) were found positive for granzyme $A$ gene expression. This frequency is $\sim 6$ times higher in the IL2R-negative population A. About $30 \%$ granzyme A mRNA-positive cells are associated with the CD3+ phenotype (populations C/D). The distribution of cells transcribing the granzyme $B$ gene is similar, as the lowest frequency of positive cells was found in population B. About $2.5 \%$ granzyme B-expressing cells are found in each of the two remaining DN subsets (Table 3 ). Cells transcribing the IL2R gene are mainly observed in population $B$, where IL2R is expressed on the surface. However, few cells containing IL2R mRNA are also found in population $\mathrm{A}$ and in the $\mathrm{CD} 3+\mathrm{DN}$ subset (C/D), both of which do not express IL2R protein on their surface (Table 3 ).

\section{Discussion}

In situ hybridizations with radiolabeled RNA probes revealed the expression of genes in the thymus which are preferentially induced in mature cytotoxic cells, i.e. ganzyme $A$ and granzyme B. However, considerable differences of gene expression in the thymus and in cell-mediated cytotoxic reactions in the periphery were observed: in particular, granzyme B-expressing thymocytes are -10 times less frequent than granzyme A mRNA-containing cells. This is in marked contrast to the situation observed in peripheral cytotoxic cells involved in the rejection of a $\mathrm{MHC}$ mismatched graft or in the clearance of liver cells infected with lymphotropic choriomeningitis virus $(8,9)$. During these reactions, granzyme B-expressing cells are present in equal or even higher numbers than granzyme A-positive cells. Expression of the granzyme $A$ and granzyme $B$ genes in the thymus was almost exclusively observed in DN, i.e. in cells which are not cytolytic unless being activated. These observations imply that the expression of the two genes in the thymus is differentially regulated and that the two serine esterases are not strictly associated with cytotoxicity. Furthermore, the absence of detectable perforin mRNA in thymocytes may imply that the function of granzymes is largely if not completely independent of perforin.

About $1.5 \%$ of granzyme A mRNA-positive cells are observed among mature, CD3-expressing CD4-CD8+ thymocytes. This corresponds with the reported frequency of granzyme Aexpressing CD8 $+\mathrm{T}$ cells in the spleen (8). The CD3CD4-CD8+ population, which may represent a transient stage between DN and CD4+CD8 + thymocytes (24), contains - 20\% granzyme A-expressing cells. Most likely these thymocytes were excluded from the CD4-CD8 + thymocyte preparation when sorting total thymocytes since they are CD8duil. This would explain the discrepancy in the percentages of granzyme Aexpressing cells when looking at total CD4-CD8+ thymocytes in Table 1 compared to the total of the two CD4-CD8+ subsets. Generally, a slight discrepancy was found between the percentage of specific mRNA-positive cells in unfractionated thymocytes and in the total of all sorted thymocyte subpopulations. Most likely, the mRNA is slowly degraded during the prolonged staining and isolation procedure and is finally undetectable by in situ hybridization in thymocytes with a low expression level of a particular gene.

Therefore we analyzed the expression of the IL2R, which is expressed at the protein level on an early DN subset (population B) (23). Immunostaining and in situ hybridization on thymus sections revealed a similar pattern of positive cells for IL2R mRNA and surface protein, respectively. IL2R mRNA-positive cells are predominantly IL2R surface protein positive. Differences between IL2R gene and protein expression are likely to reflect the in vivo situation of transient gene activation and surface expression. It may also be due in part to differences in the half-life of protein and transcript which becomes increasingly apparent with prolonged isolation procedures.

Granzyme A expression in mature cytotoxic cells can be induced by antigen in combination with lymphokines $(26,27)$ or by culturing in the presence of IL2 (28). Both activation mechanisms are unlikely to be operative in immature DN IL2RCD3 - thymocytes since these cells do not express TCR and activation of the granzyme $A$ (and $B$ ) gene does not correlate with expression of the IL2R on both mRNA and protein level. Furthermore, we did not find evidence for IL2 gene transcription with in situ hybridization on thymus sections (W. Held and C.Mueller, unpublished data).

Based on the observed high percentage of granzyme Aexpressing cells in population $A$ and their predominant blast-like morphology, we suggest that granzyme $A$ expression corresponds to cells in cycle rather than to a particular phenotype. 
This is true for population A, where $50 \%$ of the cells are in S + $\mathrm{G}_{2}+\mathrm{M}$ phase of the cell cycle $(22,29)$ and $48 \%$ of the cells express the gene for granzyme A. In the IL2R-expressing DN subset (population B) both the number of cycling and granzyme A-expressing cells are similarly reduced compared to population A. The CD3- CD4-CD8+ subpopulation represents another rapidly cycling thymocyte population which again contains a large portion of granzyme A-positive cells. The current view of intrathymic differentiation suggests a lineage from population $B$ to population A to CD3- CD4-CD8+ cycling cells to double positive, i.e. CD4+CD8+, thymocytes $(24,29)$. Thus, the granzyme A gene is induced during the early differentiation steps of thymocytes and the number of cells expressing this gene parallels the portion of cells in cycle. However, at present we do not have further evidence to confirm the relation between cell cycle status and granzyme A expression at the single cell level.

The precise function of the serine protease granzyme $A$ in the thymus as well as in CTL in the periphery is not clear. The function for granzyme $A$ in the thymus is most likely different from an involvement in cytotoxic reactions since DN thymocytes are not cytolytic per se. One could think of an involvement of granzymes in the degradation of extracellular matrix proteins (7), thus facilitating intrathymic migration of thymocytes during their differentiation. Detection of granzyme proteins, their intracellular localization, and substrate specificity in the thymus should provide further information about the precise role of granzymes in both thymocytes and CTL.

It is tempting to speculate that expression of granzymes in the thymus represents an early, perhaps transient event in $T$ cell differentiation, eventually leading to functional $T$ cells with inducible cytotoxic activity. At present, it is not clear whether all DN thymocytes express granzyme $A$ at some point during their maturation or whether cells expressing granzymes represent distinct thymic subsets, characterizing distinct differentiation lineages. In vitro differentiation and in vivo reconstitution assays with granzyme-positive and -negative thymocyte subsets may become useful to determine the functional properties and in vivo differentiation potential of thymocytes expressing granzymes.

\section{Acknowledgements}

We wish to thank $C$. Knabenhans and P.Zaech for FACS analysis and $N$. Jeanguenat and $T$. Périnat-Frey for expert technical assistance. This work was supported in part by a grant from the Swiss National Science Foundation to C.M.

\section{Abbreviations}

$\begin{array}{ll}\text { BLT } & N-\alpha \text {-benzyloxy-carbonyl-L-lysine thiobenzylester } \\ \text { CTL } & \text { cytotoxic T lymphocyte(s) } \\ \text { DN } & \text { double negative thymocytes } \\ \text { FACS } & \text { fluorescence-activated cell sorter } \\ \text { FITC } & \text { fluoroisothiocyanate } \\ \text { IL2 } & \text { interleukin 2 } \\ \text { IL2R } & \text { interleukin } 2 \text { receptor } \\ \text { NK } & \text { natural killer }\end{array}$

\section{References}

1 Masson, D. and Tschopp, J. 1987. A family of serine esterases in lytic granules of cytolytic T lymphocytes. Cell 49:679.

2 Henkart, P. A. 1985. Mechanisms of lymphocyte-mediated cytotoxicity. Annu. Rev. Immunol. 3:31.
3 Podack, E. R., Young, J. D.-E., and Cohn, Z. A. 1985. Isolation and biochemical and functional characterization of perforin 1 from cytolytic T cell granules. Proc. Natl. Acad. Sci. USA 82:8629.

4 Chang, T. W. and Eisen, H. N. 1980. Effects of N-tosyl-L-lysylchloromethylketone on the activity of cytotoxic T lymphocytes. $J$. Immunol. 124:1028.

5 Redelman, D. and Hudig, D. 1980. The mechanisms of cell-mediated cytotoxicity. I. Killing by murine cytotoxic T lymphocytes requires cell surface thiols and activated proteases. J. Immunol. 124:870.

6 Simon, M. M., Hoschuetzky, H., Fruth, U., Simon, H. G., and Kramer, M. D. 1986. Purification and characterization of a $T$ cell specific serine proteinase (TSP-1) from cloned cytolytic T lymphocytes. EMBO J. 5:3267.

7 Kramer, M. D. and Simon, M. M. 1987. Are proteinases functional molecules of T lymphocytes? Immunol. Today 8:140.

8 Mueller, C., Gershenfeld, H. K., Lobe, C. G., Okada, C. Y., Bleackley, R. C., and Weissman, I. L. 1988. A high proportion of T lymphocytes that infiltrate $\mathrm{H}$-2-incompatible heart allografts in vivo express genes encoding cytotoxic cell-specific serine proteases, but do not express the Mel-14-defined lymph node homing receptor. $J$. Exp. Med. 167:1124.

9 Mueller, C., Kaegi, D., Aebischer, T., Odermatt, B., Held, W., Podack, E. R., Zinkernagel, R. M., and Hengartner, H. 1989. Detection of perforin and granzyme A mRNAs in infiltrating cells during infection of mice with lymphocytic choriomeningitis virus. Eur. J. Immunol. 19:1253.

10 Kramer, M. D., Fruth, U., Simon, H. G., and Simon, M. M. 1989. Expression of cytoplasmic granules with $T$ cell-associated serine proteinase- 1 activity in Ly-2 $+(\mathrm{CD} 8+)$ T lymphocytes responding to lymphocytic choriomeningitis virus in vivo. Eur. J. Immunol. 19:151.

11 Young, L. H. Y., Klavinskis, L. S., Oldstone, M. B. A., and Young, J. D.-E. 1989. In vivo expression of perforin by $\mathrm{CD} 8+$ lymphocytes during an acute viral infection. J. Exp. Med. 169:2173.

12 MacDonald, $H$. R., Howe, R. C., Pedrazzini, T., Lees, R, K., Budd, R. C., Schneider, R., Liao, N. S., Zinkernagel, R. M. Louis, J. A., Raulet, D. H., Hengartner, H., and Miescher, G. 1988 T-cell lineages, repertoire selection and tolerance induction. Immunol. Rev. 104:157.

13 Lowrey, D. M., Aebischer, T., Olsen, K. J., Lichtenfeld, M., Rupp, F., Hengartner, $H_{\text {., }}$ and Podack, E. R. 1989. Cloning and analysis of murine perforin 1 (P1) CDNA, a component of cytolytic T-cell granules with homology to C9. Proc. Natl. Acad. Sci. USA 86:247.

14 Gershenfeld, H. K. and Weissman, I. L. 1986. Cloning of a cDNA for a $T$ cell-specific serine protease from a cytotoxic T lymphocyte. Science 232:854.

15 Brunet, J. F., Dosseto, M., Denizot, F., Mattei, M. G., Clark, W. R. Haqqi, T. H., Ferrier, P., Nabholz, M., Schmitt-Verhulst, A. M., Luciani, M. F., and Golstein, P. 1986. The inducible cytotoxic T lymphocyte-associated gene transcript CTLA-1 sequence and gene localization to mouse chromosome 14. Nature 322:268.

16 Pasternack, M. S. and Eisen, H. N. 1985. A novel serine esterase expressed by cytotoxic T lymphocytes. Nature 314:743

17 Young, J. D. E., Hengartner, H., Podack, E. R., and Cohn, Z. A. 1986. Purification and characterization of a cytolytic pore-forming protein from granules of cloned lymphocytes with natural killer activity. Cell 44:849.

18 Lobe, C. G., Finlay, B. B., Paranchych, W., Paetkau, V. H., and Bleackley, R. C. 1986. Novel serine proteases encoded by two cytotoxic T lymphocyte-specific genes. Science 232:858.

19 Miller, J., Malek, T. R., Leonard, W. J., Greene, W. C., Shevach, E. M., and Germain, R. N. 1985. Nucleotide sequence and expression of a mouse interleukin 2 receptor CDNA. J. Immunol. 134:4212.

20 Golstein, P., Goridis, C., Schmitt-Verhulst, A. M., Hayot, B., Pierres, A., van Agthoven, A., Kaufmann, Y., Eshar, Z., and Pierres, M. 1982. Lymphoid cell surface interaction structures detected using cytolysis inhibiting monoclonal antibodies. Immunol. Rev. 68:5.

21 Dialynas, D. P., Wilde, D. B., Marrak, P., Pierres, A., Well, K. A. Havran, W. Otten, G., Loken, M. R., Pierres, M., Kappler, J., and Fitch, F. W. 1983. Characterization of the murine antigenic determi. nant, designated L3T4a, recognized by a monoclonal antibody GK1.5: expression of L3T4a by functional T cell clones appears to correlate primarily with class II MHC antigen-reactivity. Immunol. Rev. 74:29. 22 Howe, R. C. and MacDonald, H. R. 1988. Heterogeneity of immature 
62 Expression of serine protease genes in the thymus

(Lyt2-/L3T4-) thymocytes. Identification of 4 major phenotypically distinct subsets differing in cell cycle status and in vitro activation requirements. J. Immunol. 140:1047.

23 Ceredig, R., Lowenthal, J. W., Nabholz, M., and MacDonald, H. R. 1985. Expression of interleukin-2 receptors as a differentiation marker on intrathymic stem cells. Nature $314: 98$.

24 MacDonald, H. R., Budd, R. C., and Howe, R. C. 1988. A CD3subset of $\mathrm{CD} 4-8+$ thymocytes: a rapidly cycling intermediate in the generation of CD4+8+ cells. Eur. J. Immunol. 18:519.

25 Fowlkes, B. J., Edison, L., Mathieson, B. J., and Chused, T. M. 1985. Early $\mathrm{T}$ lymphocytes. Differentiation in vivo of adult intrathymic precursor cells. J. Exp. Med. 162:802.

26 Simon, M. M., Fruth, U., Simon, H. G., and Kramer, M. D. 1986. A specific serine proteinase is inducible in Lyt-2 ${ }^{+}, \mathrm{L} 3 \mathrm{~T} 4^{-}$and Lyt-2L3T4 + T cells in vitro but is mainly associated with Lyt-2 +, L3T4- effector cells in vivo. Eur. J. Immunol. 16:1559.

27 Garcia-Sanz, J. A., Plaetinck, G., Velotti, F., Masson, D., Tschopp, J., MacDonald, H. R., and Nabholz, M. 1987. Perforin is present only in normal activated Lyt2 + T lymphocytes and not in L3T4 + cells, but the serine protease granzyme $\mathrm{A}$ is made by both subsets. EMBO J. 6:933.

28 Manyak, C. L., Norton, G. P., Lobe, C. G., Bleackley, R. C. Gershenfeld, H. K., Weissman, I. L., Kumar, V., Sigal, N. H., and Koo, C. K. 1989. IL-2 induces expression of serine protease enzymes and genes in natural killer and nonspecific T killer cells. J. Immunol. 142:3707.

29 Pearse, M., Wu, L., Egerton, M., Wilson, A., Shortman, K., and Scollay, R. 1989. A murine early thymocyte developmental sequence is marked by transient expression of the interleukin 2 receptor. Proc. Natl. Acad. Sci. USA 86:1614. 